\title{
MORE SUBLATTICES OF THE LATTICE OF LOCAL CLONES
}

\author{
MICHAEL PINSKER
}

\begin{abstract}
We investigate the complexity of the lattice of local clones over a countably infinite base set. In particular, we prove that this lattice contains all algebraic lattices with at most countably many compact elements as complete sublattices, but that the class of lattices embeddable into the local clone lattice is strictly larger than that: For example, the lattice $M_{2} \omega$ is a sublattice of the local clone lattice.
\end{abstract}

\section{LOCAL CLONES}

1.1. Defining local clones. Fix a countably infinite base set $X$, and denote for all $n \geq 1$ the set $X^{X^{n}}=\left\{f: X^{n} \rightarrow X\right\}$ of $n$-ary operations on $X$ by $\mathscr{O}^{(n)}$. Then the union $\mathscr{O}:=\bigcup_{n>1} \mathscr{O}^{(n)}$ is the set of all finitary operations on $X$. A clone $\mathscr{C}$ is a subset of $\mathscr{O}$ satisfying the following two properties:

- $\mathscr{C}$ contains all projections, i.e., for all $1 \leq k \leq n$ the operation $\pi_{k}^{n} \in \mathscr{O}^{(n)}$ defined by $\pi_{k}^{n}\left(x_{1}, \ldots, x_{n}\right)=x_{k}$, and

- $\mathscr{C}$ is closed under composition, i.e., whenever $f \in \mathscr{C}$ is $n$-ary and $g_{1}, \ldots, g_{n} \in$ $\mathscr{C}$ are $m$-ary, then the operation $f\left(g_{1}, \ldots, g_{n}\right) \in \mathscr{O}^{(m)}$ defined by

$$
\left(x_{1}, \ldots, x_{m}\right) \mapsto f\left(g_{1}\left(x_{1}, \ldots, x_{m}\right), \ldots, g_{n}\left(x_{1}, \ldots, x_{m}\right)\right)
$$

also is an element of $\mathscr{C}$.

Since arbitrary intersections of clones are again clones, the set of all clones on $X$, equipped with the order of inclusion, forms a complete lattice $\mathrm{Cl}(X)$. In this paper, we are not interested in all clones of $\mathrm{Cl}(X)$, but only in clones which satisfy an additional topological closure property: Equip $X$ with the discrete topology, and $\mathscr{O}^{(n)}=X^{X^{n}}$ with the corresponding product topology (Tychonoff topology), for every $n \geq 1$. A clone $\mathscr{C}$ is called locally closed or just local iff each of its $n$-ary fragments $\mathscr{C} \cap \mathscr{O}^{(n)}$ is a closed subset of $\mathscr{O}^{(n)}$. Equivalently, a clone $\mathscr{C}$ is local iff it satisfies the following interpolation property:

For all $n \geq 1$ and all $g \in \mathscr{O}^{(n)}$, if for all finite $A \subseteq X^{n}$ there exists

an $n$-ary $f \in \mathscr{C}$ which agrees with $g$ on $A$, then $g \in \mathscr{C}$.

Again, taking the set of all local clones on $X$, and ordering them according to set-theoretical inclusion, one obtains a complete lattice, which we denote by $\mathrm{Cl}_{\text {loc }}(X)$ : This is because intersections of clones are clones, and because arbitrary intersections of closed sets are closed. We are interested in the structure of $\mathrm{Cl}_{\text {loc }}(X)$, in particular in how complicated it is as a lattice.

2000 Mathematics Subject Classification. Primary 08A40; secondary 08A05.

Key words and phrases. clone; local closure; algebraic lattice; embedding.

The author is grateful for support through Erwin Schrödinger Fellowship J2742-N18 of the Austrian Science Fund (FWF). 
Before we start our investigations, we give an alternative description of local clones which will be useful. Let $f \in \mathscr{O}^{(n)}$ and let $\rho \subseteq X^{m}$ be a relation. We say that $f$ preserves $\rho$ iff $f\left(r_{1}, \ldots, r_{n}\right) \in \rho$ whenever $r_{1}, \ldots, r_{n} \in \rho$; here, the $m$-tuple $f\left(r_{1}, \ldots, r_{n}\right)$ is calculated componentwise, i.e., it is the $m$-tuple whose $i$-th component is obtained by applying $f$ to the $n$-tuple consisting of the $i$-th components of the tuples $r_{1}, \ldots, r_{n}$. For a set of relations $\mathscr{R}$, we write $\operatorname{Pol}(\mathscr{R})$ for the set of those operations in $\mathscr{O}$ which preserve all $\rho \in \mathscr{R}$. The operations in $\operatorname{Pol}(\mathscr{R})$ are called polymorphisms of $\mathscr{R}$. The following is due to Rom77, see also the textbook Sze86].

Theorem 1. $\operatorname{Pol}(\mathscr{R})$ is a local clone for all sets of relations $\mathscr{R}$. Moreover, every local clone is of this form.

Similarly, for an operation $f \in \mathscr{O}^{(n)}$ and a relation $\rho \subseteq X^{m}$, we say that $\rho$ is invariant under $f$ iff $f$ preserves $\rho$. Given a set of operations $\mathscr{F} \subseteq \mathscr{O}$, we write $\operatorname{Inv}(\mathscr{F})$ for the set of all relations which are invariant under all $f \in \mathscr{F}$. Since arbitrary intersections of local clones are local clones again, the mapping on the power set of $\mathscr{O}$ which assigns to every set of operations $\mathscr{F} \subseteq \mathscr{O}$ the smallest local clone $\langle\mathscr{F}\rangle_{l o c}$ containing $\mathscr{F}$ is a closure operator, the closed elements of which are exactly the local clones. Using the operators Pol and Inv which connect operations and relations, one obtains the following well-known alternative for describing this operator (confer Rom77] or [Sze86]).

Theorem 2. Let $\mathscr{F} \subseteq \mathscr{O}$. Then $\langle\mathscr{F}\rangle_{l o c}=\operatorname{Pol} \operatorname{Inv}(\mathscr{F})$.

As already mentioned, the aim of this paper is to investigate the structure of the local clone lattice. So far, this lattice has been studied only sporadically, e.g. in RS82, RS84. There, the emphasis was put on finding local completeness criteria for sets of operations $\mathscr{F} \subseteq \mathscr{O}$, i.e., on how to decide whether or not $\langle\mathscr{F}\rangle_{l o c}=\mathscr{O}$. Only very recently has the importance of the local clone lattice to problems from model theory and theoretical computer science been revealed:

1.2. The use of local clones. Let $\Gamma=(X, \mathscr{R})$ be a countably infinite structure; that is, $X$ is a countably infinite base set and $\mathscr{R}$ is a set of finitary relations on $X$. Consider the expansion $\Gamma^{\prime}$ of $\Gamma$ by all relations which are first-order definable from $\Gamma$. More precisely, $\Gamma^{\prime}$ has $X$ as its base set and its relations $\mathscr{R}^{\prime}$ consist of all finitary relations which can be defined from relations in $\mathscr{R}$ using first-order formulas. A reduct of $\Gamma^{\prime}$ is a structure $\Delta=(X, \mathscr{D})$, where $\mathscr{D} \subseteq \mathscr{R}^{\prime}$. We also call $\Delta$ a reduct of $\Gamma$, which essentially amounts to saying that we expect our structure $\Gamma$ to be closed under first-order definitions. Clearly, the set of reducts of $\Gamma$ is in one-to-one correspondence with the power set of $\mathscr{R}^{\prime}$, and therefore not of much interest as a partial order. However, it might be more reasonable to consider such reducts up to, say, first-order interdefinability. That is, we may consider two reducts $\Delta_{1}=\left(X, \mathscr{D}_{1}\right)$ and $\Delta_{2}=\left(X, \mathscr{D}_{2}\right)$ the same iff their first-order expansions coincide, or equivalently iff all relations of $\Delta_{1}$ are first-order definable in $\Delta_{2}$ and vice-versa.

In 1976, P. J. Cameron Cam76 showed that there are exactly five reducts of $(\mathbb{Q},<)$ up to first-order interdefinability. Recently, M. Junker and M. Ziegler gave a new proof of this fact, and established that $(\mathbb{Q},<, a)$, the expansion of $(\mathbb{Q},<)$ by a constant $a$, has 116 reducts [JZ08]. S. Thomas proved that the first-order theory of the random graph also has exactly five reducts, up to first-order interdefinability Tho91]. 
These examples have in common that the structures under consideration are $\omega$-categorical, i.e., their first-order theories determine their countable models up to isomorphism. This is no coincidence: For, given an $\omega$-categorical structure $\Gamma$, its reducts up to first-order interdefinability are in one-to-one correspondence with the locally closed permutation groups which contain the automorphism group of $\Gamma$, providing a tool for describing such reducts (confer [Cam90]).

A natural variant of these concepts is to consider reducts up to primitive positive interdefinability. That is, we consider two reducts $\Delta_{1}, \Delta_{2}$ of $\Gamma$ the same iff their expansions by all relations which are definable from each of the structures by primitive positive formulas coincide. (A first-order formula is called primitive positive iff it is of the form $\exists \bar{x}\left(\phi_{1} \wedge \cdots \wedge \phi_{l}\right)$ for atomic formulas $\phi_{1}, \ldots, \phi_{l}$.) It turns out that for $\omega$-categorical structures $\Gamma$, the local clones containing all automorphisms of $\Gamma$ are in one-to-one correspondence with those reducts of the first-order expansion of $\Gamma$ which are closed under primitive positive definitions. This recent connection, which relies on a theorem from [BN06, has already been exploited in $\mathrm{BCP}$, where the reducts of $(X,=)$, the structure whose only relation is the equality, have been classified using this method. It turns out that despite the simplicity of this structure, the lattice of its reducts is quite complex, and in particular has the size of the continuum.

We mention in passing that distinguishing relational structures up to primitive positive interdefinability, and therefore understanding the structure of $\mathrm{Cl}_{\text {loc }}(X)$, has recently gained significant importance in theoretical computer science, more precisely for what is known as the Constraint Satisfaction Problem; see BKJ05, Bod04, or also the introduction in $\mathrm{BCP}$.

1.3. Main results of this paper. In this paper, which is the journal version of a shorter article which appeared in the conference proceedings of the ROGICS'08 conference Pin08, we are concerned with Problem V of the survey paper GP08, which asks which sublattices $\mathrm{Cl}_{\text {loc }}(X)$ contains. We prove that every algebraic lattice with countably many compact elements is a complete sublattice of $\mathrm{Cl}_{\text {loc }}(X)$ (Theorem 5). We also show that $\mathrm{Cl}_{\text {loc }}(X)$ is, with respect to size, not too far from such lattices as it as a join-preserving embedding into an algebraic lattice with countably many compacts (Theorem 8). All this is done in Section 2. In Section 3, we prove that the lattice $M_{2}$ embeds into $\mathrm{Cl}_{\text {loc }}(X)$ (Theorem 14), thereby providing the first example of a sublattice of $\mathrm{Cl}_{\text {loc }}(X)$ which does not embed into any algebraic lattice with countably many compacts.

We also pose a series of open problems, one in Section 2 (Problem[11), and three more in an own open problems section, Section 4 (Problems 20, 21, 22).

1.4. Acknowledgement. I am grateful to Marina Semenova for her critical remarks which forced me to find correct proofs of the theorems.

\section{Algebraic lattices and the local Clone lattice}

We start our investigations by observing that whereas the lattice $\mathrm{Cl}(X)$ of all (not necessarily local) clones over $X$ is algebraic, it has been discovered recently in GP08 that the local clone lattice $\mathrm{Cl}_{\text {loc }}(X)$ is far from being so; in fact, the following has been shown.

Proposition 3. The only compact element in the lattice $\mathrm{Cl}_{\mathrm{loc}}(X)$ is the clone of projections. 
We remark that it follows from the proof of the preceding proposition given in GP08, that $\mathrm{Cl}_{\text {loc }}(X)$ is not even upper continuous.

We now turn to sublattices of $\mathrm{Cl}_{\text {loc }}(X)$. The following is a first easy observation which tells us that there is practically no hope that $\mathrm{Cl}_{\text {loc }}(X)$ can ever be fully described, since it is believed that already the clone lattice over a three-element set is too complex to be completely understood.

Proposition 4. Let $\mathrm{Cl}(A)$ be the lattice of all clones over a finite set $A$. Then $\mathrm{Cl}(A)$ is an isomorphic copy of an interval of $\mathrm{Cl}_{\mathrm{loc}}(X)$.

Proof. Assume without loss of generality that $A \subseteq X$. Assign to every operation $f\left(x_{1}, \ldots, x_{n}\right)$ on $A$ a set of $n$-ary operations $\mathscr{S}_{f} \subseteq \mathscr{O}^{(n)}$ on $X$ as follows: An operation $g \in \mathscr{O}^{(n)}$ is an element of $\mathscr{S}_{f}$ iff $g$ agrees with $f$ on $A^{n}$. Let $\sigma$ map every clone $\mathscr{C}$ on the base set $A$ to the set $\bigcup\left\{\mathscr{S}_{f}: f \in \mathscr{C}\right\}$. Then the following hold:

(1) For every clone $\mathscr{C}$ on $A, \sigma(\mathscr{C})$ is a local clone on $X$.

(2) $\sigma$ maps the clone of all operations on $A$ to $\operatorname{Pol}(\{A\})$.

(3) All local clones (in fact: all clones) which contain $\sigma(\{f: f$ is a projection on $A\}$ ) (i.e., which contain the local clone on $X$ which, via $\sigma$, corresponds to the clone of projections on $A$ ) and which are contained in $\operatorname{Pol}(\{A\})$ are of the form $\sigma(\mathscr{C})$ for some clone $\mathscr{C}$ on $A$.

(4) $\sigma$ is one-to-one, and both $\sigma$ and its inverse are order preserving.

(1) and (2) are easy verifications and left to the reader. To see (3), let $\mathscr{D}$ be any clone in the mentioned interval, and denote by $\mathscr{C}$ the set of all restrictions of operations in $\mathscr{D}$ to appropriate powers of $A$. Since $\mathscr{D} \subseteq \operatorname{Pol}(\{A\})$, all such restrictions are operations on $A$, and since $\mathscr{D}$ is closed under composition and contains all projections, so does $\mathscr{C}$. Thus, $\mathscr{C}$ is a clone on $A$. We claim $\mathscr{D}=\sigma(\mathscr{C})$. By the definitions of $\mathscr{C}$ and $\sigma$, we have that $\sigma(\mathscr{C})$ clearly contains $\mathscr{D}$. To see the less obvious inclusion, let $f \in \sigma(\mathscr{C})$ be arbitrary, say of arity $m$. The restriction of $f$ to $A^{m}$ is an element of $\mathscr{C}$, hence there exists an $m$-ary $f^{\prime} \in \mathscr{D}$ which has the same restriction to $A^{m}$ as $f$. Define $s\left(x_{1}, \ldots, x_{m}, y\right) \in \mathscr{O}^{(m+1)}$ by

$$
s\left(x_{1}, \ldots, x_{m}, y\right)= \begin{cases}y & , \text { if }\left(x_{1}, \ldots, x_{m}\right) \in A^{m} \\ f\left(x_{1}, \ldots, x_{m}\right) & , \text { otherwise. }\end{cases}
$$

Since $s$ behaves on $A^{m+1}$ like the projection onto the last coordinate, and since $\mathscr{D}$ contains $\sigma(\{f: f$ is a projection on $A\})$, we infer $s \in \mathscr{D}$. But $f\left(x_{1}, \ldots, x_{m}\right)=$ $s\left(x_{1}, \ldots, x_{m}, f^{\prime}\left(x_{1}, \ldots, x_{m}\right)\right)$, proving $f \in \mathscr{D}$.

(4) is an immediate consequence of the definitions.

It is known that all countable products of finite lattices embed into the clone lattice over a four-element set Bul94, so by the preceding proposition they also embed into $\mathrm{Cl}_{\text {loc }}(X)$. However, there are quite simple countable lattices which do not embed into the clone lattice over any finite set: The lattice $M_{\omega}$ consisting of a countably infinite antichain plus a smallest and a greatest element is an example Bul93. We shall see now that the class of lattices embeddable into $\mathrm{Cl}_{\text {loc }}(X)$ properly contains the class of lattices embeddable into the clone lattice over a finite set. In fact, the structure of $\mathrm{Cl}_{\mathrm{loc}}(X)$ is at least as complicated as the structure of any algebraic lattice with $\aleph_{0}$ compact elements. Before we prove this, observe that similarly to the local clones, the set of locally closed (that is: topologically closed in the space of all permutations on $X$ ) permutation groups on $X$ forms a complete 
lattice with respect to inclusion; denote this lattice by $\operatorname{Gr}_{\text {loc }}(X)$. Moreover, the set of locally closed (that is: closed in the space $X^{X}$, where $X$ is discrete) transformation monoids on $X$ forms a complete lattice with respect to inclusion, which we denote by $\operatorname{Mon}_{\text {loc }}(X)$. Note that the elements of $\operatorname{Mon}_{\text {loc }}(X)$ are precisely the objects of the form $\mathscr{C} \cap \mathscr{O}^{(1)}$, where $\mathscr{C} \in \mathrm{Cl}_{\text {loc }}(X)$.

Theorem 5. Every algebraic lattice with a countable number of compact elements is a complete sublattice of $\mathrm{Cl}_{\text {loc }}(X)$. In fact, every such lattice is a complete sublattice of $\mathrm{Gr}_{\mathrm{loc}}(X)$ and of $\mathrm{Mon}_{\mathrm{loc}}(X)$.

It is clear that the lattice $\operatorname{Mon}_{\text {loc }}(X)$ of locally closed transformation monoids embeds completely into $\mathrm{Cl}_{\text {loc }}(X)$ via the assignment which sends every local monoid to the local clone it generates. Local clones arising in this way will contain only operations which depend on at most one variable: In fact, the operations of such a local clone are exactly the functions of the monoid, with (possibly) some fictitious variables added. Therefore, the statement of the theorem about the lattice $\operatorname{Mon}_{\text {loc }}(X)$ implies the statement about $\mathrm{Cl}_{\text {loc }}(X)$.

To prove Theorem [5, we cite the following deep theorem from [Tim89].

Theorem 6. Every algebraic lattice with a countable number of compact elements is isomorphic to an interval in the subgroup lattice of a countable group.

Proof of Theorem 5. We prove the statement about $\operatorname{Gr}_{\text {loc }}(X)$. Let $\mathfrak{L}$ be the algebraic lattice to be embedded into $\operatorname{Gr}_{\text {loc }}(X)$. Let $\mathfrak{X}=(X,+,-, 0)$ be the group provided by Theorem 6 . Let $\left[\mathfrak{G}_{1}, \mathfrak{G}_{2}\right]$ be the interval in the subgroup lattice of $\mathfrak{X}$ that $\mathfrak{L}$ is isomorphic to. We will assign to every group in the interval its Cayley representation as a group of permutations on $X$ : That is, for every $a \in X$, define a unary operation $f_{a} \in \mathscr{O}^{(1)}$ by $f_{a}(x)=a+x$. Clearly, we have $f_{a}\left(f_{b}(x)\right)=a+b+x=f_{a+b}(x)$ for all $a, b \in X$. Define a mapping $\mu:\left[\mathfrak{G}_{1}, \mathfrak{G}_{2}\right] \rightarrow \operatorname{Gr}_{\text {loc }}(X)$ sending every group $\mathfrak{H}=(H,+,-, 0)$ in the interval to $\mathscr{C}_{H}:=\left\{f_{a}: a \in H\right\}$. It is easy to see (and folklore) that the $\mathscr{C}_{H}$ are permutation groups; we only have to check that they are locally closed. To see this, let $f \in \mathscr{O}^{(1)}$ be an element of the topological (local) closure of $\mathscr{C}_{H}$ in the full symmetric group on $X$. We claim $f \in \mathscr{C}_{H}$. Indeed, observe that $f$ agrees with some $f_{a} \in \mathscr{C}_{H}$ on the finite set $\{0\} \subseteq X$. Suppose that there is $b \in X$ such that $f(b) \neq f_{a}(b)=a+b$. Then take any $f_{c} \in \mathscr{C}_{H}$ such that $f$ and $f_{c}$ agree on $\{0, b\}$. But then $c=f_{c}(0)=f(0)=f_{a}(0)=a$, and thus $f(b)=f_{c}(b)=f_{a}(b) \neq f(b)$, an obvious contradiction. Hence, $f=f_{a} \in \mathscr{C}_{H}$ and we are done.

With the explicit description of the $\mathscr{C}_{H}$ and given that they are indeed closed permutation groups, a straightforward check shows that $\mu$ preserves arbitrary meets and joins.

The proof for the embedding into $\operatorname{Mon}_{\text {loc }}(X)$ is identical. By the discussion above the statement about $\mathrm{Cl}_{\mathrm{loc}}(X)$ follows.

Since in particular, $\mathrm{Cl}_{\text {loc }}(X)$ contains $M_{\omega}$ as a sublattice, and since according to Bul93, $M_{\omega}$ is not a sublattice of the clone lattice over any finite set, we have the following corollary to Theorem 5 .

Corollary 7. $\mathrm{Cl}_{\mathrm{loc}}(X)$ does not embed into the clone lattice over any finite set.

Observe also that Theorem 5 is a strengthening of Proposition 4 in so far as the clone lattice over a finite set is an example of an algebraic lattice with countably 
many compact elements. However, in that proposition we obtain an embedding as an interval, not just as a complete sublattice.

What about other lattices, i.e., lattices which are more complicated or larger than algebraic lattices with countably many compact elements? We will now find a restriction on which lattices can be sublattices of $\mathrm{Cl}_{\mathrm{loc}}(X)$.

A partial clone of finite operations on $X$ is a set of partial operations of finite domain on $X$ which contains all restrictions of the projections to finite domains and which is closed under composition. A straightforward verification shows that the set of partial clones of finite operations on $X$ forms a complete algebraic lattice $\mathrm{Cl}_{\text {fpart }}(X)$, the compact elements of which are precisely the finitely generated partial clones; in particular, the number of compact elements of $\mathrm{Cl}_{\text {fpart }}(X)$ is countable.

Theorem 8. The mapping $\sigma$ from $\mathrm{Cl}_{\text {loc }}(X)$ into $\mathrm{Cl}_{\text {fpart }}(X)$ which sends every $\mathscr{C} \in$ $\mathrm{Cl}_{\mathrm{loc}}(X)$ to the partial clone of all restrictions of its operations to finite domains is one-to-one and preserves arbitrary joins.

Proof. It is obvious that $\sigma(\mathscr{C})$ is a partial clone of finite operations, for all local (in fact: also non-local) clones $\mathscr{C}$.

Let $\mathscr{C}, \mathscr{D} \in \mathrm{Cl}_{\text {loc }}(X)$ be distinct. Say without loss of generality that there is an $n$-ary $f \in \mathscr{C} \backslash \mathscr{D}$; then since $\mathscr{D}$ is locally closed, there exists some finite set $A \subseteq X^{n}$ such that there is no $g \in \mathscr{D}$ which agrees with $f$ on $A$. The restriction of $f$ to $A$ then witnesses that $\sigma(\mathscr{C}) \neq \sigma(\mathscr{D})$.

We show that $\sigma(\mathscr{C}) \vee \sigma(\mathscr{D})=\sigma(\mathscr{C} \vee \mathscr{D})$; the proof for arbitrary joins works the same way. It follows directly from the definition of $\sigma$ that it is order-preserving. Thus, $\sigma(\mathscr{C} \vee \mathscr{D})$ contains both $\sigma(\mathscr{C})$ and $\sigma(\mathscr{D})$ and hence also their join. Now let $f \in \sigma(\mathscr{C}) \vee \sigma(\mathscr{D})$. This means that it is a composition of partial operations in $\sigma(\mathscr{C}) \cup \sigma(\mathscr{D})$. All partial operations used in this composition have extensions to operations in $\mathscr{C}$ or $\mathscr{D}$, and if we compose these extensions in the same way as the partial operations, we obtain an operation in $\mathscr{C} \vee \mathscr{D}$ which agrees with $f$ on the domain of the latter. Whence, $f \in \sigma(\mathscr{C} \vee \mathscr{D})$.

Corollary 9. $\mathrm{Cl}_{\mathrm{loc}}(X)$ embeds as a suborder into the power set of $\omega$. In particular, the size of $\mathrm{Cl}_{\mathrm{loc}}(X)$ is $2^{\aleph_{0}}$, and $\mathrm{Cl}_{\mathrm{loc}}(X)$ does not contain any uncountable ascending or descending chains.

Proof. The number of partial operations with finite domain on $X$ is countable; therefore, partial clones of finite operations can be considered as subsets of $\omega$, which proves the first statement. In particular, $\mathrm{Cl}_{\text {loc }}(X)$ cannot have more than $2^{\aleph_{0}}$ elements, and the fact that all algebraic lattices with at most $\aleph_{0}$ compact elements embed into $\mathrm{Cl}_{\text {loc }}(X)$ shows that it must contain at least $2^{\aleph_{0}}$ elements. The third statement is an immediate consequence of the first one.

It also follows from Theorem 8 that for all sets of local clones $S \subseteq \mathrm{Cl}_{\text {loc }}(X)$, there exists a countable $S^{\prime} \subseteq S$ such that $\bigvee S=\bigvee S^{\prime}$

It might be interesting to observe at this point that $\mathrm{Cl}_{\text {fpart }}(X)$ is universal for the class of algebraic lattices with countably many compact elements: Denote by $\operatorname{Mon}_{\text {fpart }}(X)$ the lattice of all sets of finite partial unary operations on $X$ which are closed under composition, and which contain all restrictions of the identity. In other words, the elements of $\operatorname{Mon}_{\text {fpart }}(X)$ are precisely the unary fragments of the elements of $\mathrm{Cl}_{\text {fpart }}(X)$. As before with local monoids and local clones, $\operatorname{Mon}_{\text {fpart }}(X)$ 
embeds naturally into $\mathrm{Cl}_{\text {fpart }}(X)$ by adding fictitious variables to the partial operations of an element of $\operatorname{Mon}_{\text {fpart }}(X)$. We have:

Proposition 10. Every algebraic lattice with a countable number of compact elements is a complete sublattice of $\mathrm{Cl}_{\text {fpart }}(X)$. In fact, every such lattice is a complete sublattice of $\mathrm{Mon}_{\mathrm{fpart}}(X)$.

Proof. Let $\mathfrak{L}$ be the lattice to be embedded, and embed it into $\operatorname{Mon}_{\text {loc }}(X)$ as in the proof of Theorem 5 via the mapping $\mu$ of that proof. Then apply the mapping $\sigma$ from Theorem 8 to the local monoids thus obtained. This obviously gives us a join-preserving mapping from $\mathfrak{L}$ into $\operatorname{Mon}_{\text {fpart }}(X)$. We claim that $\sigma$, restricted to monoids in the image of $\mu$, preserves arbitrary meets: Let $\mathscr{C}_{H}, \mathscr{C}_{K}$ be two such monoids. Clearly, $\sigma\left(\mathscr{C}_{H} \cap \mathscr{C}_{K}\right)$ is contained in $\sigma\left(\mathscr{C}_{H}\right) \cap \sigma\left(\mathscr{C}_{K}\right)$. Now let $p \in \sigma\left(\mathscr{C}_{H}\right) \cap$ $\sigma\left(\mathscr{C}_{K}\right)$. Then, using the notation from the proof of Theorem 5 there exist a finite set $A \subseteq X, a \in H$ and $b \in K$ such that $p=f_{a} \Upsilon_{A}=f_{b} \Upsilon_{A}$. We may assume that $A$ is non-void; pick $c \in A$. We have $p(c)=f_{a}(c)=a+c=f_{b}(c)=b+c$. Hence, $a=b$, so $f_{a}=f_{b} \in \mathscr{C}_{H} \cap \mathscr{C}_{K}$, and $p \in \sigma\left(\mathscr{C}_{H} \cap \mathscr{C}_{K}\right)$. Larger meets work the same way, and thus we have a complete lattice embedding of $\mathfrak{L}$ into $\operatorname{Mon}_{\text {fpart }}(X)$.

Until today, no other restriction to embeddings into $\mathrm{Cl}_{\text {loc }}(X)$ except for Theorem 8 is known, and we ask:

Problem 11. Does every lattice which has a complete join-embedding into an algebraic lattice with countably many compacts have a lattice embedding into $\mathrm{Cl}_{\mathrm{loc}}(X)$ ?

It seems, however, difficult to embed even the simplest lattices which are not covered by Theorem 5 into $\mathrm{Cl}_{\text {loc }}(X)$. In the conference version of this paper, Pin08, the following problem was posed:

Does the lattice $M_{2^{\omega}}$, which consists of an antichain of length $2^{\omega}$ plus a smallest and a largest element, embed into $\mathrm{Cl}_{\text {loc }}(X)$ ?

We will give an affirmative answer to this problem in Section 3. This is the first example of a lattice which embeds into $\mathrm{Cl}_{\text {loc }}(X)$ but not into any algebraic lattice with countably many compacts (for the latter statement, see the proof of Corollary 13).

It is much easier to see the following:

Proposition 12. There exist a join-preserving embedding as well as a meet-preserving embedding of $M_{2} \omega$ into $\mathrm{Cl}_{\mathrm{loc}}(X)$.

Proof. Denote by 0 and 1 the smallest and the largest element of $M_{2^{\omega}}$, respectively, and enumerate the elements of its antichain by $\left(a_{i}\right)_{i \in 2^{\omega}}$.

We first construct a join-preserving embedding. Enumerate the non-empty proper subsets of $X$ by $\left(A_{i}\right)_{i \in 2^{\omega}}$. Consider the mapping $\sigma$ which sends 0 to the clone of projections, 1 to $\mathscr{O}$, and every $a_{i}$ to $\operatorname{Pol}\left(\left\{A_{i}\right\}\right)$. Now it is well-known (see [RS84]) that for any non-empty proper subset $A$ of $X, \operatorname{Pol}(\{A\})$ is covered by $\mathscr{O}$, i.e. there exist no local (in fact even no global) clones between $\operatorname{Pol}(\{A\})$ and $\mathscr{O}$. Hence, we have that $\sigma\left(a_{i}\right) \vee \sigma\left(a_{j}\right)=\left\langle\operatorname{Pol}\left(\left\{A_{i}\right\}\right) \cup \operatorname{Pol}\left(\left\{A_{j}\right\}\right)\right\rangle_{l o c}=\mathscr{O}=\sigma(1)$ for all $i \neq j$. Since clearly $\sigma\left(a_{i}\right)$ contains $\sigma(0)$ for all $i \in 2^{\omega}$, the mapping $\sigma$ indeed preserves joins.

To construct a meet-preserving embedding, fix any distinct $a, b \in X$ and define for every non-empty subset $A$ of $X \backslash\{a, b\}$ an operation $f_{A} \in \mathscr{O}^{(1)}$ by

$$
f_{A}(x)= \begin{cases}a, & \text { if } x \in A \\ b, & \text { otherwise }\end{cases}
$$


Enumerate the non-empty subsets of $X \backslash\{a, b\}$ by $\left(B_{i}: i \in 2^{\omega}\right)$. Denote the constant unary operation with value $b$ by $c_{b}$. Let the embedding $\sigma$ map 0 to $\left\langle\left\{c_{b}\right\}\right\rangle_{\text {loc }}$, for all $i \in 2^{\omega}$ map $a_{i}$ to $\left\langle\left\{f_{B_{i}}\right\}\right\rangle_{l o c}$, and let it map 1 to $\mathscr{O}$. One readily checks that $\sigma\left(a_{i}\right)=\left\langle\left\{f_{B_{i}}\right\}\right\rangle_{\text {loc }}$ contains only projections and, up to fictitious variables, the operations $f_{B_{i}}$ and $c_{b}$. Therefore, for $i \neq j$ we have $\sigma\left(a_{i}\right) \wedge \sigma\left(a_{j}\right)=\left\langle\left\{c_{b}\right\}\right\rangle_{l o c}=\sigma(0)$. Since clearly $\sigma\left(a_{i}\right) \subseteq \sigma(1)=\mathscr{O}$ for all $i \in 2^{\omega}$, we conclude that $\sigma$ does indeed preserve meets.

Simple as the preceding proposition is, it still shows us as a consequence that Theorem 5 is not optimal.

Corollary 13. $\mathrm{Cl}_{\text {loc }}(X)$ is not embeddable into any algebraic lattice with countably many compact elements.

Proof. It is well-known and easy to check (confer also [CD73]) that any algebraic lattice $\mathfrak{L}$ with countably many compact elements can be represented as the subalgebra lattice of an algebra over the base set $\omega$. The meet in the subalgebra lattice $\mathfrak{L}$ is just the set-theoretical intersection. Now there is certainly no uncountable family of subsets of $\omega$ with the property that any two distinct members of this family have the same intersection $D$; for the union of such a family would have to be uncountable. Consequently, $\mathfrak{L}$ cannot have $M_{2} \omega$ as a meet-subsemilattice. But $\mathrm{Cl}_{\text {loc }}(X)$ has, hence $\mathfrak{L}$ cannot have $\mathrm{Cl}_{\text {loc }}(X)$ as a sublattice.

Observe that this corollary is a strengthening of Corollary 7 since the clone lattice over a finite set is an algebraic lattice with countably many compact elements.

We conclude this section by remarking that the lattice $\mathrm{Cl}(X)$ of all (not necessarily local) clones on $X$ is infinitely more complicated than $\mathrm{Cl}_{\text {loc }}(X)$ : It contains all algebraic lattices with at most $2^{\aleph_{0}}$ compact elements, and in particular all lattices of size continuum (including $\mathrm{Cl}_{\text {loc }}(X)$ ), as complete sublattices Pin07.

\section{How to Embed $M_{2^{\omega}}$ INTO $\mathrm{Cl}_{\mathrm{loc}}(X)$}

This section is devoted to the proof of the following theorem.

Theorem 14. $M_{2^{\omega}}$ is isomorphic to a sublattice of $\mathrm{Cl}_{\mathrm{loc}}(X)$.

Write $X=A \cup \dot{\cup} \cup \dot{\cup}\{\infty$, with both $A$ and $B$ infinite. Without loss of generality assume $B=2^{<\omega}$, the set of all finite 0-1-sequences. For every infinite 0-1-sequence $\alpha \in 2^{\omega}$, write $B_{\alpha}:=\left\{\alpha\left\lceil_{n}: n<\omega\right\}\right.$. Clearly, the $B_{\alpha}$ form an almost disjoint family of infinite subsets of $B$, that is, $B_{\alpha} \cap B_{\beta}$ is finite whenever $\alpha, \beta \in 2^{\omega}$ are distinct. For all $\alpha \in 2^{\omega}$, set $\mathscr{G}_{\alpha} \subseteq \mathscr{O}^{(1)}$ to consist of all functions $f \in \mathscr{O}^{(1)}$ satisfying the following two properties:

- $f$ maps $\{\infty\} \cup B$ to $\infty$, and

- $f$ maps $A$ injectively into $B_{\alpha}$.

For $c, d \in B$, we write $c \perp d$ iff none of the two elements is an initial segment of the other, i.e. iff there is no $\alpha$ such that $c, d \in B_{\alpha}$. If $C, D \subseteq B$, we write $C \perp D$ iff $c \perp d$ for all $(c, d) \in C \times D$. For a function $\sigma$, write $\operatorname{dom}(\sigma)$ and $\operatorname{ran}(\sigma)$ for the domain and the range of $\sigma$, respectively. Now for all functions $\sigma$ with the properties

- $\operatorname{dom}(\sigma)=C \times D$ for some finite $C, D \subseteq B$ with $C \perp D$, and

- $\operatorname{ran}(\sigma) \subseteq B$, and

- $\sigma$ is injective, 
define an operation $m^{\sigma} \in \mathscr{O}^{(2)}$ by

$$
m^{\sigma}(x, y):= \begin{cases}\infty & , x=\infty \vee y=\infty \\ y & , x \in A \wedge y \in B \\ \sigma(x, y) & ,(x, y) \in \operatorname{dom}(\sigma) \\ x & , \text { otherwise. }\end{cases}
$$

In words, $m^{\sigma}$ does the following: If one of its arguments equals $\infty$, then it returns $\infty$. If one of the arguments is in $A$ and the other one in $B$, then it returns the one in $B$. If both arguments are in $B$ and $\sigma$ is defined for $(x, y)$, then $m^{\sigma}(x, y)=\sigma(x, y)$. Otherwise, $m^{\sigma}$ returns the first argument.

Denote by $\mathscr{M}$ the set of all such operations $m^{\sigma}$, together with the constant function with value $\infty$, which we denote by $\infty$ as well. For all $\alpha \in 2^{\omega}$, set $\mathscr{D}_{\alpha}:=$ $\left\langle\mathscr{M} \cup \mathscr{G}_{\alpha}\right\rangle$, which is to denote the normal clone closure of $\mathscr{M} \cup \mathscr{G}_{\alpha}$, i.e., the set of all operations which can be written as a term of the operations in $\mathscr{M} \cup \mathscr{G}_{\alpha}$ and the projections. Set moreover $\mathscr{C}_{\alpha}:=\left\langle\mathscr{M} \cup \mathscr{G}_{\alpha}\right\rangle_{\text {loc }}$ (the topological closure of $\mathscr{D}_{\alpha}$ ), for all $\alpha \in 2^{\omega}$. Finally, set $\mathscr{C}:=\left\langle\bigcup_{\alpha \in 2^{\omega}} \mathscr{C}_{\alpha}\right\rangle_{l o c}$.

We now aim at proving $\left\langle\mathscr{C}_{\alpha} \cup \mathscr{C}_{\beta}\right\rangle_{l o c}=\mathscr{C}$ and $\mathscr{C}_{\alpha} \cap \mathscr{C}_{\beta}=\langle\mathscr{M}\rangle_{l o c}$, for all distinct $\alpha, \beta \in 2^{\omega}$, which clearly implies our theorem.

Lemma 15. Let $m \in \mathscr{M}$, and $f, g \in \mathscr{G}_{\alpha}$ for some $\alpha \in 2^{\omega}$. Then the following equations hold:

$$
m(x, x)=x \quad \text { and } \quad m(f(x), x)=m(x, f(x))=m(f(x), g(x))=f(x) .
$$

Proof. We leave the straightforward verification to the reader.

Denote the identity operation on $X$ by id.

Lemma 16. Let $f \in \mathscr{C}_{\alpha}^{(1)}$. Then $f \in \mathscr{G}_{\alpha}$, or $f=\infty$, or $f=$ id.

Proof. We first prove the statement for all $f \in \mathscr{D}_{\alpha}^{(1)}$, using induction over terms. The beginning is trivial, so let $f=g(t)$, with $g \in \mathscr{G}_{\alpha} \cup\{$ id $\}$ and $t \in \mathscr{D}_{\alpha}^{(1)}$ satisfying the induction hypothesis. There is nothing to show if $t$ is the identity or $\infty$. If $t \in \mathscr{G}_{\alpha}$, then also $f \in \mathscr{G}_{\alpha}$ if $g$ is the identity; otherwise, $g \in \mathscr{G}_{\alpha}$ and $f=\infty$. Now assume $f=m(s, t)$, with $m \in \mathscr{M}$ and $s, t$ satisfying the induction hypothesis. Then the preceding lemma immediately implies our assertion.

Now with this description of $\mathscr{D}_{\alpha}^{(1)}$ it is easy to check that $\mathscr{D}_{\alpha}^{(1)}$ is locally closed. Hence $\mathscr{C}_{\alpha}^{(1)}=\mathscr{D}_{\alpha}^{(1)}$, proving the lemma.

Lemma 17. Let $t\left(x_{1}, \ldots, x_{n}\right) \in \mathscr{D}_{\alpha}$, and assume that $t$ has a representation as a term over $\mathscr{G}_{\alpha} \cup \mathscr{M}$ (without projections!) which uses at least one symbol from $\mathscr{G}_{\alpha}$. Then either $t=\infty$, or $t(x, \ldots, x) \in \mathscr{G}_{\alpha}$.

Proof. We use induction over the complexity of $t$. The beginning is trivial, so write $t=g(s)$, where $g \in \mathscr{G}_{\alpha}$ and $s$ satisfies the induction hypothesis. Assume first that $s$ does not contain any symbol from $\mathscr{G}_{\alpha}$; then $s(x, \ldots, x)=x$ by Lemma 15 and a standard induction, implying $t(x, \ldots, x)=g(s(x, \ldots, x))=g(x) \in \mathscr{G}_{\alpha}$. If on the other hand $s$ does contain a symbol from $\mathscr{G}_{\alpha}$, then the range of $s$ is contained in $B \cup\{\infty\}$, as is easily verified by a straightforward induction using Lemma 15 . Hence, $g(s)=\infty$. Now write $t=m(r, s)$, with $m \in \mathscr{M}$ and $r, s$ satisfying the induction hypothesis. Then our assertion follows from Lemma 15 and the definition of the operations in $\mathscr{M}$. 
Lemma 18. Let $\alpha, \beta \in 2^{\omega}$ be distinct. Then $\mathscr{C}_{\alpha} \cap \mathscr{C}_{\beta}=\langle\mathscr{M}\rangle_{\text {loc }}$.

Proof. It suffices to show $\mathscr{C}_{\alpha} \cap \mathscr{C}_{\beta} \subseteq\langle\mathscr{M}\rangle_{\text {loc }}$. To see this, let $t\left(x_{1}, \ldots, x_{n}\right) \in$ $\mathscr{C}_{\alpha} \cap \mathscr{C}_{\beta}$, and suppose there is a finite set $F \subseteq X$ such that no operation in $\langle\mathscr{M}\rangle$ (normal clone closure) agrees with $t$ on $F^{n}$. By expanding $F$, we may assume that $|F \cap A|>\left|B_{\alpha} \cap B_{\beta}\right|$ (since the latter set is finite). Let $s$ be a term over $\mathscr{G}_{\alpha} \cup \mathscr{M}$ which interpolates $t$ on $F^{n}$. By our assumption, there appears some function from $\mathscr{G}_{\alpha}$ in $s$. Then by Lemma 17, $s(x, \ldots, x) \in \mathscr{G}_{\alpha}$. Therefore, also $t(x, \ldots, x)$ behaves like an operation from $\mathscr{G}_{\alpha}$ on $F$, and in particular on $F \cap A$. Hence, it maps $F \cap A$ injectively into $B_{\alpha}$. Now the same argument shows us that $t(x, \ldots, x)$ maps $F \cap A$ injectively into $B_{\beta}$, hence it maps $F \cap A$ injectively into $B_{\alpha} \cap B_{\beta}$, in contradiction with the size of these sets.

Lemma 19. Let $\alpha, \beta \in 2^{\omega}$ be distinct. Then $\left\langle\mathscr{C}_{\alpha} \cup \mathscr{C}_{\beta}\right\rangle_{\text {loc }}=\mathscr{C}$.

Proof. Let $\gamma \in 2^{\omega}, \gamma \notin\{\alpha, \beta\}$ be arbitrary, and consider any $h \in \mathscr{G}_{\gamma}$. It suffices to show that $h \in\left\langle\mathscr{C}_{\alpha} \cup \mathscr{C}_{\beta}\right\rangle_{\text {loc }}$. So let $F \subseteq X$ be finite. We have to find an operation in $\left\langle\mathscr{C}_{\alpha} \cup \mathscr{C}_{\beta}\right\rangle$ which agrees with $h$ on $F$. Assume for the moment that $F \subseteq A$. Pick any $C \subseteq B_{\alpha}, D \subseteq B_{\beta}$ with $|C|=|D|=|F|$ and such that $C \perp D$. Pick $f \in \mathscr{G}_{\alpha}$ mapping $F$ onto $C$, and $g \in \mathscr{G}_{\beta}$ mapping $F$ onto $D$. Then $(f, g): X \rightarrow X^{2}$ maps $F$ injectively into $C \times D$. Thus there exists $\sigma: C \times D \rightarrow B_{\gamma}$ such that $\sigma(f(x), g(x))=h(x)$ for all $x \in F$. Now $m^{\sigma}(f(x), g(x))=\sigma(f(x), g(x))=h(x)$ for all $x \in F$, so $m^{\sigma}(f(x), g(x)) \in\left\langle\mathscr{C}_{\alpha} \cup \mathscr{C}_{\beta}\right\rangle$ agrees with $h$ on $F$.

For the case where $F$ is not a subset of $A$, one constructs the interpolation for $F^{\prime}:=F \cap A$. Then observe that the operation $m^{\sigma}(f(x), g(x))$ constructed sends all $b \in B \cup\{\infty\}$ to $\infty$, thus behaving like $h$ outside $A$ anyway.

Lemmas 18 and 19 clearly prove Theorem 14

\section{Open PROBlems}

Suprisingly, the method of the previous section seems to be hard to generalize: For example, we do not know:

Problem 20. Does every lattice of finite height which has cardinality $2^{\aleph_{0}}$ embed into $\mathrm{Cl}_{\mathrm{loc}}(X)$ ?

When proving that all algebraic lattices with countably many compacts embed into $\mathrm{Cl}_{\text {loc }}(X)$, we in fact embedded them into $\operatorname{Mon}_{\text {loc }}(X)$ (Theorem 5 ). The same could be true with $M_{2}$ (which we do not believe, though):

Problem 21. Does $M_{2^{\omega}}$ embed into $\operatorname{Mon}_{\text {loc }}(X)$ ? Does it embed into $\operatorname{Gr}_{\text {loc }}(X)$ ?

In theory, even the following could hold:

Problem 22. Does $\mathrm{Cl}_{\text {loc }}(X)$ embed into $\mathrm{Mon}_{\text {loc }}(X)$ ? Does it embed into $\mathrm{Gr}_{\text {loc }}(X)$ ?

\section{REFERENCES}

[BCP] M. Bodirsky, H. Chen, and M. Pinsker. The reducts of equality up to primitive positive interdefinability. Journal of Symbolic Logic. To appear. Preprint available from http://arxiv.org/abs/0810.2270

[BKJ05] A. Bulatov, A. Krokhin, and P. G. Jeavons. Classifying the complexity of constraints using finite algebras. SIAM Journal on Computing, 34:720-742, 2005.

[BN06] M. Bodirsky and J. Nešetrril. Constraint satisfaction with countable homogeneous templates. Journal of Logic and Computation, 16(3):359-373, 2006. 
[Bod04] M. Bodirsky. Constraint satisfaction with infinite domains. Dissertation, HumboldtUniversität zu Berlin, 2004.

[Bul93] A. Bulatov. Identities in lattices of closed classes. Discrete Mathematics and Applications, 3(6):601-609, 1993.

[Bul94] A. Bulatov. Finite sublattices in the lattice of clones. Algebra and Logic, 33(5):287-306, 1994.

[Cam76] P. J. Cameron. Transitivity of permutation groups on unordered sets. Mathematische Zeitschrift, 148:127-139, 1976.

[Cam90] P. J. Cameron. Oligomorphic permutation groups, volume 152 of London Mathematical Society Lecture Note Series. Cambridge University Press, Cambridge, 1990.

[CD73] P. Crawley and R. P. Dilworth. Algebraic theory of lattices. Prentice-Hall, 1973.

[GP08] M. Goldstern and M. Pinsker. A survey of clones on infinite sets. Algebra Universalis, 59:365-403, 2008.

[JZ08] M. Junker and M. Ziegler. The 116 reducts of $(\mathbb{Q},<, a)$. Journal of Symbolic Logic, 73(3):861-884, 2008.

[Pin07] M. Pinsker. Algebraic lattices are complete sublattices of the clone lattice on an infinite set. Fundamenta Mathematicae, 195(1):1-10, 2007.

[Pin08] M. Pinsker. Sublattices of the lattice of local clones. In Proceedings of the ROGICS'08 conference, pages 80-87, 2008 .

[Rom77] B. A. Romov. Galois correspondence between iterative post algebras and relations on infinite sets. Cybernetics, 3:377-379, 1977.

[RS82] I. G. Rosenberg and D. Schweigert. Locally maximal clones. Elektron. Informationsverarb. Kybernet., 18(7-8):389-401, 1982. in German, Russian summary.

[RS84] I. G. Rosenberg and L. Szabó. Local completeness. I. Algebra Universalis, 18(3):308-326, 1984.

[Sze86] Á. Szendrei. Clones in universal algebra. Les Presses de L'Université de Montréal, 1986.

[Tho91] S. Thomas. Reducts of the random graph. Journal of Symbolic Logic, 56(1):176-181, 1991.

[Tům89] J. Tůma. Intervals in subgroup lattices of infinite groups. Journal of Algebra, 125(2):367399, 1989.

E-mail address: marula@gmx.at

$U R L:$ http://dmg.tuwien.ac.at/pinsker/

Laboratoire de Mathématiques Nicolas Oresme, CNRS UMR 6139, Université de Caen, 14032 Caen Cedex, France 